\title{
Un cómputo de eras melkita del siglo XIII contenido en el 'Códice árabe sinaítico 445': edición, traducción y estudio
}

\author{
A 13th century Melkite calculation of eras contained in the 'Codex Sinaitic \\ Arabic 445': edition, translation and study \\ Juan Pedro MONFERRER-SALA \\ Universidad de Córdoba \\ ff1mosaj@uco.es
}

Recibido: enero 2013

Aceptado: marzo 2013

\section{RESUMEN}

En el presente trabajo ofrecemos la edición y traducción del 'Códice sinaítico 445' del Monasterio de Santa Catalina, Monte Sinaí, precedido de un estudio filológico del texto y seguido del análisis de aquellos aspectos de interés que presenta la redacción.

Palabras clave: cómputo, eras, melkita, Sinaí, siglo XIII, Códice árabe sinaítico 445.

\begin{abstract}
In this paper we offer the edition and translation of 'Codex Sinaitic 445' in the Monastery of Saint Catherine, Mount Sinai. Edition and translation are preceded by a philological study of the text. At the same time, an analysis of those aspects of interest present in the text has been added.
\end{abstract}

Keywords: calculation, eras, Melkite, Sinai, 13th century, Codex Sinaitic Arabic 445.

SUMARIO: 0. Introducción, 1. Estudio paleográfico y lingüístico, a) Onomástica, b) Topónimos, c) Préstamos y neologismos, d) Terminología cronológica, 2. Edición diplomática del texto árabe, 3. Notas al texto. 


\section{INTRODUCCIÓN}

Los dos fols. $\left(77^{\mathrm{r}}-77^{\mathrm{v}}\right)$ del texto que analizamos y editamos en este trabajo forman parte del códice sinaítico 445 (Sin. ar. 445). Se trata de un códice misceláneo del siglo XIII, concretamente del año 1233, cuyo soporte de escritura es el papel y cuenta con unas medidas de $17 \times 12.5 \mathrm{~cm} .{ }^{1}$

El texto ofrece los cálculos cronológicos que median entre narraciones, acontecimientos y personajes del texto bíblico. No se trata de una práctica sin importancia, sino todo lo contrario, pues dentro de todo el complejo entramado de la cronología ( $\operatorname{ta}$ rīh $h$ bíblica en este tipo de textos sus redactores perseguían situar el flujo cronológico en relación con la figura de Jesús. ${ }^{2}$

Esta tipología textual, por lo demás, no se encuentra muy alejada de la que ofrecen algunos textos de Qumrān (4Q559), donde el objetivo de los cómputos es calcular la llegada del Mesías. ${ }^{3}$ De hecho, las profecías escatológicas fueron las que animaron las prácticas cronológicas en modalidades diversas, ${ }^{4}$ de acuerdo con los variados métodos de enseñanza que ejercitaron los maestros en la antigüedad clásica. ${ }^{5}$

En el caso del presente texto, se trata de un autor melkita que utiliza el sistema conocido como Annus Mundi o era de la creación, que en árabe es referido de varios modos: ta'rīh al-hilqī, li-Ādam o kawn al-ālam. ${ }^{6}$ Dado que se creía que la venida de Jesús tendría lugar a mediados del sexto milenio, se convino que el punto de partida de la era sería el año 5500 a.C., pese a que entre los dos principales métodos de cómputo mediaban 17 años entre sí: así el sistema alejandrino o era de Anniano daba comienzo el 25 de marzo del año 5492 a.C., en tanto que el bizantino

${ }^{1}$ ATIYA, Aziz Suryal. The Arabic Manuscripts of Mount Sinai. Baltimore, 1955, 13 (n 445); cf. KAMIL, Murad. Catalogue of all manuscripts in the Monastery of St. Catharine on Mount Sinai. Wiesbaden, 1970, $39\left(\mathrm{n}^{\circ} 493\right)$.

${ }^{2}$ Cf. MOSSHAMMER. Alden A. The Easter Computus and the Origins of the Christian Era. New York, 2008, 413. Sobre esta obra, véase la reseña de Philipp E. NOTHAFT en Byzantinische Zeitschrift 1 (2009), pp. 255-258. Véase, además, Ph. E. NOTHAFT. Dating the Passion: The Life of Jesus and the Emergence of Scientific Chronology (200-1600), Leiden: Brill, 2011.

${ }^{3}$ EISENMAN, Robert H. y WISE, Michael, Dead Sea Scrolls Uncovered. The First Complete Translation and Interpretation of 50 Key Documents Withheld for over 35 Years. New York, 1993, 9293. Cf. LANDES, Richard. «Millenialism», en The Oxford Handbook of New Religious Movements, ed. by James R. LEWIS. New York, 2004, 341.

4 "Eras", en A Guide to the Postclassical World, ed. G.W. BOWERSOCK, Peter BROWN y Oleg GRABAR. Cambridge MA,1999, 429.

${ }^{5}$ SAMUEL, Alan E. Greek and Roman Chronology. Calendars and Years in Classical Antiquity. München 1972.

${ }^{6}$ Cf. GACEK, Adam. Arabic Manuscripts. A Vademecum for Readers, Leiden. 2009, 60.

${ }^{7}$ The Chronography of George Synkellos: A Byzantine Chronicle of Universal History from the Creation. Translated with Introduction and Notes by William ADLER and Paul TUFFIN. New York, 2002, xxxii. 
comenzaba el 1 de septiembre del año 5509 a.C. Un tercer sistema en discordia, el de Julio Africano daba comienzo el año 5502 a.C. ${ }^{8}$

Como podremos comprobar al final del presente trabajo, en el cuadro que ofrecemos con las correspondencias entre las referencias bíblicas y los cómputos cronológicos del tiempo transcurrido entre cada una de las dos referencias, junto con los dos cómputos globales, este autor melkita ofrece una equivalencia plena con el punto de partida de la era en el año 5500. ${ }^{9}$

Por otro lado, este sistema de cómputo de eras aplicado a la historia bíblica en su recepción cristiana oriental forma parte de un proceso más amplio de uso de los diversos sistemas cronológicos y de datación utilizados por los cristianos orientales en función del tipo de comunidad eclesiástica a la que perteneciera el autor o el copista de la obra: siriaca oriental, siriaca occidental, copta (calcedoniana o siria ortodoxa), maronita o melkita, que es la comunidad que utilizó este sistema tanto en textos compuestos en árabe como en siriaco. ${ }^{10}$

\section{ESTUDIO PALEOGRÁFICO Y LINGÜÍSTICO}

El tipo grafológico del que se sirve el copista es el sistema nashī utilizado en un importante número de copias conservadas en el scriptorium de Mār Katirīnah en el Monte Sinaí. El texto exhibe numerosas deficiencias en la realización de los grafemas y sus correspondientes diacríticos, ${ }^{11}$ al tiempo que presenta una serie de fenómenos lingüísticos propios del registro lingüístico conocido como 'árabe medio': v.gr. el fenómeno de hipercorrección morfológica $-\bar{l} n<-i y y i n$ que presenta aliskandarīn $<$ al-iskandiriyyīn $\left(3^{\mathrm{v}}, 4^{\mathrm{v}}\right) .^{12}$

Como en otros casos, estas irregularidades a las que acabamos de referirnos son la consecuencia de dos causas: a) la rapidez con la que el copista ha plasmado el texto, que, ya total ya parcialmente, le ha llevado a omitir con frecuencia los diacríticos de las consonantes que los portan; y b) a las interferencias de fenómenos

\footnotetext{
${ }^{8}$ GRUMEL, V. La chronologie. Paris, 1958, 92-94, 97. Véase además, para el caso de Julio Africano, MOSSHAMMER, Alden A. The Easter Computus, 418.

${ }^{9}$ Theophilus of Edessa's Chronicle and the Circulation of Historical Knowledge in Late Antiquity and Early Islam. Translated with an introduction and notes by Robert G. HOYLAND. Liverpool, 2011, 40. Cf. PUMMER, Reinhard. Early Christian Authors on Samaritans and Samaritanism. Tübingen, 2002, 409.

${ }^{10}$ HATCH, William H.P. An Album of Dated Syriac Manuscripts. Boston MA, 1946, 19. Véase también BROCK, Sebastian. "The use of Hijra dating in Syriac manuscripts: a preliminary investigation", en Redefining Christian Identity: Cultural Interaction in the Middle East since the Rise of Islam, ed. J.J. VAN GINKEL et al. Louvain, 2005, 276, n. 3.

${ }^{11}$ BLAU, Joshua. A Grammar of Christian Arabic. Based Mainly on South-Palestinian Texts from the First Millenium. 3 vols. Louvain, 1966-67, I, 122-125 §§ 25-26.3.2. En adelante: GCA.

${ }^{12}$ BLAU, J. GCA, I, 232 § 125.
} 
lingüísticos procedentes del registro neoárabe. En el caso de a) las deficiencias ortográficas que exhibe el texto son las siguientes:

- consonantes con ausencia de diacríticos en el nivel suprasegmental:

/t/: muståān $\left(1^{\mathrm{r}}\right)$, náalum < táallum $\left(2^{\mathrm{r}}\right)$, yastadilu $<$ yastadillu $\left(4^{\mathrm{r}}\right)$, mutaqadimīn $<$ mutaqaddimīn $\left(7^{r}\right)$, tåàle $<$ ta ālà $\left(8^{r}\right)$, miyatay $<$ mi’atay $\left(9^{r}\right)$.

/t: : talatah $<$ talātata $^{t}\left(12^{\mathrm{v}}\right), \underline{\text { tamāninn }}<$ țamānīna $\left(12^{\mathrm{v}}\right)$.

/h/: tawārīh < tawārīh $\left(1^{\mathrm{r}}\right)$.

$/ \mathrm{f} /:$ : $f \hat{i}\left(7^{\mathrm{rbis}}\right)$, țüfän $\left(9^{\mathrm{r}}\right.$, frente a $10^{\mathrm{r}}$ donde ha sido realizado), alf $<$ 'alf $\left(12^{\mathrm{v}}, 13^{\mathrm{v}}\right)$.

/q/: mutaqadimīn $<$ mutaqaddimin $\left(7^{\mathrm{r}}\right)$.

$/ \mathrm{n} /$ : muståān $\left(1^{\mathrm{r}}\right)$, ilaynā < îlaynā $\left(6^{\mathrm{r}}\right)$, min $\left(7^{\mathrm{r}}, 10^{\mathrm{r}}, 13^{\mathrm{r}}, 8^{\mathrm{v}}, 10^{\mathrm{v}}, 13^{\mathrm{v}}\right)$, alfā̄n $<$ 'alfān $\left(9^{\mathrm{r}}\right)$, sanah $<\operatorname{sana}^{t}\left(9^{\mathrm{r}}, 2^{\mathrm{v}}, 5^{\mathrm{v}}, 7^{\mathrm{v}}, 9^{\mathrm{v}}, 10^{\mathrm{v}}, 11^{\mathrm{vbis}}, 12^{\mathrm{v}}\right)$, nabī $\left(1^{\mathrm{v}}\right)$, arba în $<$ 'arbaīn $\left(2^{\mathrm{v}}\right)$, al-iskandarīn < al-'iskandariyyīn $\left(4^{\mathrm{v}}, 5^{\mathrm{v}}\right)$, yūnāni $\left(6^{\mathrm{v}}\right)$, hamsīn $<$ hamsīn $\left(10^{v}\right)$, tamānīn $<$ tamāninna $\left(12^{v}\right)$.

- consonantes con ausencia de diacríticos en el nivel infrasegmental:

/b/ bi- (10r), abnā < 'abnā' $\left(13^{\mathrm{r}}\right), \operatorname{arba}^{\mathrm{e}}<\mathrm{\prime}^{\prime} \operatorname{arba}^{\mathrm{e}}\left(14^{\mathrm{r}}\right), \operatorname{nabi}\left(1^{\mathrm{v}}\right), \operatorname{subh}\left(14^{\mathrm{v}}\right)$.

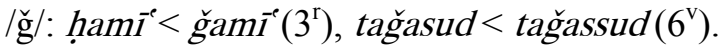

$/ \mathrm{y} /:$ ilaynā $<$ ilaynā $\left(6^{\mathrm{r}}\right)$, fi $\left(7^{\mathrm{rbis}}\right)$, mutaqadimīn $<$ mutaqaddimīn $\left(7^{\mathrm{r}}\right)$, sin̄̄n $\left(8^{\mathrm{r}}\right)$, miyatay $<$ miatay $\left(9^{\mathrm{r}}\right)$, miyah $<$ miat $\left(10^{\mathrm{r}}, 13^{\mathrm{v}}\right)$, Ibrahìm $<$ Ibrāhìm $\left(12^{\mathrm{r}}\right)$, 'alayhi $\left(12^{\mathrm{r}}, 13^{\mathrm{r}}, 2^{\mathrm{v}}, 3^{\mathrm{v}}\right)$, situmiyah $<$ sittumi'a ${ }^{t}\left(12^{\mathrm{r}}, 2^{\mathrm{v}}, 4^{\mathrm{v}}, 12^{\mathrm{v}}\right)$, Isrāyil $<$ 'Isrâ̄îl $\left(14^{\mathrm{r}}, 1^{\mathrm{v}}\right)$, arba'amiyah < 'arba'ami'at ${ }^{t}\left(14^{\mathrm{r}}\right)$, nabi $\left(1^{\mathrm{v}}, 3^{\mathrm{v}}\right)$, al-iskandarīn $<$ al-'iskandariyyīn $\left(4^{\mathrm{v}}, 5^{\vee}\right)$, Fìlibus < Fīlibbus $\left(4^{\mathrm{v}}\right)$, yūnānī $\left(6^{\mathrm{v}}\right)$, hamsīn $<$ hamsīn $\left(10^{\mathrm{v}}\right)$, tamānīn $<$ tamāninna $\left(12^{\mathrm{v}}\right)$, amīn $<$ 'amīn $\left(14^{\mathrm{v}}\right)$.

En el uso de la preposición ilà, la alif maqșūrah ${ }^{13}$ adopta los dos diacríticos de la $y \bar{a}^{\circ}$, con el consabido cambio $/ \overline{\mathrm{e}} /</ \overline{\mathrm{a}} /:$ ile $(* i l y)<$ illà $\left(1^{\mathrm{v}}, 3^{\mathrm{v}}\right)$; el mismo fenómeno se da en tåāle < tåālà $\left(8^{\mathrm{r}}\right)$. En cuanto al fenómeno de la alif maqșürah en lugar de la $y a \bar{a}$ 'éste se aprecia en la preposición $f \grave{l}\left(7^{\mathrm{r}}\right)$, banà $<\operatorname{banī}\left(14^{\mathrm{r}}, 1^{\mathrm{v}}\right)$.

En el nivel suprasegmental, el uso de la šaddah o tašdīd ${ }^{14}$ es inexistente en todo el texto: amā $<$ 'ammā $\left(2^{\mathrm{r}}\right)$, na'alum $<$ ta'allum $\left(2^{\mathrm{r}}\right)$, an $<$ 'anna $\left(2^{\mathrm{r}}\right)$, quwāt $<$ quwwāt $\left(3^{\mathrm{r}}\right)$, yastadilu $<$ yastadillu $\left(4^{\mathrm{r}}\right)$, yamuru $<$ yamurru $\left(4^{\mathrm{r}}\right)$, al-ayām $<$ al'ayyām $\left(5^{\mathrm{r}}\right)$, mutaqadimīn $<$ mutaqaddimīn $\left(7^{\mathrm{r}}\right)$, 'amā $<$ 'ammā $\left(7^{\mathrm{r}}\right)$, anahu $<$ 'annahu

${ }^{13}$ BLAU, J., GCA, 81-83 $\S 10.1-10.3$; HOPKINS, Simon. Studies in the Grammar of Early Arabic. Based upon papyri datable to before A.H. 300/A.D. 912. Oxford, 1984, 14-16 § 12; BENGTSSON, P.Å., Two Arabic Versions of the Book of Ruth. Text edition and language. Lund, 1995, 104 § 3.2.3; KNUTSSON, B., Studies in the Text and Language of three Syriac-Arabic Versions of the Book of Judicum with Special Reference to the Middle Arabic Elements. Introduction-Linguistic Notes-Texts. Leiden, 1974, 58-59.

${ }^{14}$ BLAU, J., GCA, 122-125 §§ 26.1-26.3.2; HOPKINS, S., Studies, 49 § 48; CORRIENTE, Federico. A grammatical sketch of the Spanish Arabic dialect bundle. Madrid, 1977, 66-67 § 3.2.1-3.2.2. 
$\left(8^{\mathrm{r}}\right)$, sitah $<$ sitta $^{t}\left(11^{\mathrm{r}}, 12^{\mathrm{v}}\right)$, Fīlibus $<$ Fīlibbus $\left(4^{\mathrm{v}}, 5^{\mathrm{v}}\right)$, tağasud $<$ tağassud $\left(6^{\mathrm{v}}\right)$, sayid $<$ sayyid $\left(6^{\mathrm{v}}, 8^{\mathrm{v}} 13^{\mathrm{v}}\right)$, muhaliṣ $<$ muhalliș $\left(6^{\mathrm{v}}\right)$, li-l-Lah $<$ li-l-Lāh $\left(14^{\mathrm{v}}\right)$.

El rasgo sociolectal característico de los textos árabes cristianos de la escritura Alah ('Dios') carente de šaddah, y en consecuencia en scriptio defectiva,${ }^{15}$ se debe al interés del copista de diferenciarlo del uso morfológico que hacen del término los autores musulmanes de la voz Allāh: Alah $<$ Allāh $\left(1^{\mathrm{r}}, 2^{\text {rbis }}, 8^{\mathrm{r}}\right)$. Lo mismo sucede con el uso de la alif maddah ${ }^{16}$ que siempre se omite: Adam $<\overline{\text { Adam }}\left(9^{\mathrm{r}}, 13^{\mathrm{v}}\right)$, ahir $<\overline{\text { ăhir }}\left(11^{\mathrm{v}}\right)$.

En el nivel vocálico se advierte ausencia de vocales breves a lo largo del texto, con la excepción de los siguientes vocablos: tabāraka $\left(8^{\mathrm{r}}\right)$, donde realiza todas las mociones breves, y en Alah, donde ha escrito la fatḥah de la lām $\left(8^{\mathrm{r}}\right)$. En algunos casos la vocal tanwīn del acusativo es marcada: ịhsānan < ịhsānan $\left(6^{\mathrm{r}}\right)$. El copista recurre a la scriptio plena en casos como al-samāwāt $\left(3^{\mathrm{r}}\right)$ y $\operatorname{Da} w \bar{u} d\left(1^{\mathrm{v}}, 3^{\mathrm{v}}\right)$, en tanto que hay presencia de scriptio defectiva en el antropónimo Ibrahìm $<$ Ibrāhìm $\left(12^{\mathrm{r}}, 13^{\mathrm{r}}\right)$ y en los numerales cardinales talatīn $\left(5^{\mathrm{v}}\right)$, talatamiyah $<$ talatamia $^{t}\left(7^{\mathrm{v}}\right)$ y talatah $<$ talatatat $^{t}\left(12^{\mathrm{v}}\right)$. En un caso la vocal larga $/ \mathbf{1} /$ ha sido reducida a breve $/ \mathrm{i} /$ por asimilación con hamzah tras alif mamdūdah: Isrāyil < 'Isrā̄îl $\left(14^{\mathrm{r}}, 1^{\mathrm{v}}\right)$. En materia

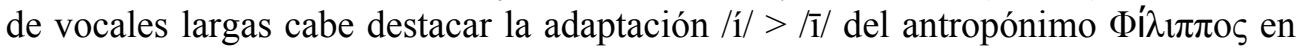
la forma árabe Fìlibbus $\left(4^{\mathrm{v}}, 5^{\mathrm{v}}\right) .^{17}$

En el nivel consonántico, ${ }^{18}$ frente al uso estable que exhibe el fonema hamzah ${ }^{19}$ en árabe clásico, en neoárabe, por el contrario, sólo es realizado en posición inicial aunque sin evidencia ortográfica, aun cuando su función fonémica independiente se haya perdido desde los primeros momentos. ${ }^{20}$ Nuestro texto omite el grafema /'/ en todas las posiciones, lo cual evidencia que la pausa articulada de la / $/$ no era perceptible en el registro utilizado por el autor o el copista, exhibiendo de este modo la pronunciación coloquial de esta consonante.

a) Inicial: amā $<$ 'ammā $\left(2^{\mathrm{r}}\right)$, an < 'anna $\left(2^{\mathrm{r}}\right)$, ilaynā $<$ ỉlaynā $\left(6^{\mathrm{rbis}}\right)$, iḥsānan

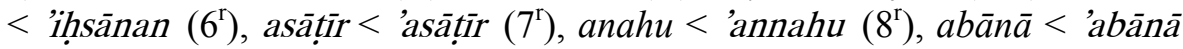
$\left(9^{\mathrm{r}}\right)$, alfān < 'alfān $\left(9^{\mathrm{r}}\right)$, ilà < ỉlà $\left(10^{\mathrm{r}}, 12^{\mathrm{r}}, 13^{\mathrm{r}}, 6^{\mathrm{v}}, 8^{\mathrm{v}}, 11^{\mathrm{v}} 14^{\mathrm{v}}\right)$, Ibrahìm $<$ 'Ibrāhīm $\left(12^{\mathrm{r}}, 13^{\mathrm{r}}\right)$, abnā < 'abnā' $\left(13^{\mathrm{r}}\right)$, Isrāyil < 'Isrā'îl $\left(14^{\mathrm{r}}, 1^{\mathrm{v}}\right)$, ard $<$ 'ard

${ }^{15}$ Cf. BROCK, Sebastian P. y HOPKINS, Simon. «A verse homily on Abraham and Sarah in Egypt: Syriac original with early Arabic translation». Le Muséon 105/1-2 (1992), 99.

${ }^{16}$ HOPKINS, S., Studies, 49 § 49i.

${ }^{17}$ Cf. MONFERRER-SALA, Juan Pedro. "Mutatio nominum. Onomástica griega en transcripción árabe”, Collectanea Cristiana Orientalia 4 (2007), 73-108.

${ }^{18}$ BLAU, J., GCA, 83-121 $\S$ 11-24.4; CANTINEAU, Jean. Études de linguistique arabe. Mémorial Jean Cantineau. Paris, 1960, 13-88; BENGTSSON, P.A. Two Arabic Versions, 108-128 §§ 3.2.5-3.2.16; KNUTSSON, B. Studies, 59-112; CORRIENTE, F. Sketch, 31-60 §§ 2.1.1-2.28.7.

${ }^{19}$ BLAU, J., GCA, 83-89 §§ 11-11.3.6.1 HOPKINS, S., Studies, 19-33 §§ 19-28; BENGTSSON, P., Studies, 108-114 §§ 3.2.5-3.2.5.3; KNUTSSON, B. Studies, 59-78; CORRIENTE, F. Sketch, 58-60 $\S \S 2.28 .1-2.28 .7$.

${ }^{20}$ BLAU, J., GCA, 83-84 §§ 11-11.1. 
(14 $)$, arba'amiyah < 'arba'ami'at $\left(14^{\mathrm{r}}\right)$, arba $<$ 'arba $^{\mathrm{e}}\left(14^{\mathrm{r}}\right)$, arba în $<$ 'arbain $\left(2^{\mathrm{v}}\right)$, alf $<$ 'alf $\left(9^{\mathrm{v}}\right)$, alf $<$ 'alf $\left(12^{\mathrm{v}}, 13^{\mathrm{v}}\right)$, amin $<$ 'amīn $\left(14^{\mathrm{v}}\right)$.

b) Medial: al-ih̆wah < al-ihhwat $\left(2^{\mathrm{r}}\right)$, al-a wām < al-'a 'wām $\left(5^{\mathrm{r}}\right)$, al-ayām $<$ al'ayyām $\left(5^{\mathrm{r}}\right)$, situmiyah $<$ sittumi'a ${ }^{t}\left(12^{\mathrm{r}}, 2^{\mathrm{v}}, 4^{\mathrm{v}}, 12^{\mathrm{v}}\right)$, Isrāyil $<$ 'Isrāāil $\left(14^{\mathrm{r}}, 1^{\mathrm{v}}\right)$, al-iskandarīn $<$ al-'iskandariyyīn $\left(4^{\mathrm{v}}, 5^{\mathrm{v}}\right)$, miya $<$ mi' $^{t}\left(9^{\mathrm{v}}, 13^{\mathrm{v}}\right)$, dāyiman $<$ däiman $\left(14^{\mathrm{v}}\right)$.

c) Final ${ }^{21}$ : binā $<$ bină $^{\prime}\left(10^{\mathrm{r}}, 11^{\mathrm{r}}\right)$, abnā $<{ }^{\prime} a b n a \bar{a}^{\prime}\left(13^{\mathrm{r}}\right)$.

Aunque debemos precisar que no todos los fenómenos son el producto de cambios fonéticos, los cambios consonánticos que presenta el texto son los siguientes:

$/ \mathrm{bb} /</ \pi \pi /:^{22}$ Fïlibbus $<\Phi$ í $1 \pi \pi \mathrm{o}\left(4^{\mathrm{v}}, 5^{\mathrm{v}}\right)$.

$/ \mathrm{t} /</ \mathrm{t} /:{ }^{23}$ tabat $<$ tabat $\left(7^{\mathrm{r}}\right)$, aunque en otras ocasiones es realizado correctamente, v.gr. talatīin $\left(5^{\vee}\right)$, talatamia ${ }^{t}\left(7^{\vee}\right)$.

$/ \mathrm{h} /</ \check{\mathrm{g}} /:^{24}$ hurūụh $<$ hururüğ $\left(13^{\mathrm{r}}\right)$.

$/ \mathrm{h} /</ \mathrm{h} /:^{25}$ tawārị̣̄ $<$ tawārīh̆ $\left(1^{\mathrm{r}}\right)$, ḥalaqa $<$ halaqa $\left(8^{\mathrm{r}}\right)$, hamsūn $<$ hamsūna $\left(12^{\mathrm{r}}\right)$, hurüḥ $<$ hurü̆ğ $\left(13^{\mathrm{r}}\right.$, aunque aparece correctamente realizado en $\left.1^{\mathrm{v}}\right)$, muḥaliṣ < muhallis $\left(6^{\mathrm{v}}\right)$, ḥamsīn $<$ hamsinn $\left(10^{\mathrm{v}}\right)$, a hị $<$ āhir $\left(11^{\mathrm{v}}\right)$, ḥams $<$ hams $\left(13^{\mathrm{vbis}}\right)$.

$/ \mathrm{d} /</ \mathrm{d} /{ }^{26}$ dikarah $<\operatorname{dikara}^{t}\left(7^{\mathrm{v}}, 8^{\mathrm{v}}\right)$, aunque ha sido realizado en las deixis hadeā $\left(1^{\mathrm{r}}\right)$ y hadihi $\left(7^{\mathrm{v}}, 8^{\mathrm{v}}\right)$.

$/ \mathrm{s} /</ \breve{\mathbf{s}} /:^{27}$ nasà $<$ naš $\left(11^{\mathrm{v}}\right)$.

${ }^{21}$ Sobre el acento en el 'árabe cristiano' antiguo y su relación con el registro magrebí antiguo, véase BLAU, J. 'Middle and Old Arabic Material for the History of Stress in Arabic'. Bulletin of the Schools of Oriental and African Studies 35/3 (1972), 476-484.

${ }^{22}$ BLAU, J., The Emergence and Linguistic Background of Judaeo-Arabic. A Study of the Origins of Middle Arabic. Jerusalem, 1981, 134; Federico CORRIENTE. Sketch, 35 § 2.2.3.; cfr. además 31$32 \S 2.1 .1$ y $34 \S 2.2 .1$; MONFERRER-SALA, J.P., «Descripción lingüística de la columna árabe del Suppl. grec $911 \mathrm{BnF}$ (año 1043)», Collectanea Christiana Orientalia 2 (2005), 114.

${ }^{23}$ BLAU, J. GCA, 106, § 12.4; 107-108 § 15.2; KNUTSSON, B., Studies, 78-94; BENGTSSON, P.Å., Two Arabic Versions, 114-119 § 3.2.6; CORRIENTE, F. Sketch, $44 \S$ 2.12.2; Joshua BLAU, Emergence, 76, 227 y 231; MONFERRER-SALA, J.P., «Descripción», Collectanea Christiana Orientalia 2 (2005), 114.

${ }^{24}$ MONFERRER-SALA, J.P., «Descripción», Collectanea Christiana Orientalia 2 (2005), 115.

${ }^{25}$ MONFERRER-SALA, J.P., «Descripción», Collectanea Christiana Orientalia 2 (2005), 116. Cf. BROCKELMANN, Carl. Grundriß der vergleichende Grammatik der semitischen Sprachen, Berlin, 1908, I, $121 \S 45$.

${ }^{26}$ BLAU, J. GCA, $108 \S 16.2$; BLAU, J. Emergence, 76, 227, 231; KNUTSSON, B. Studies, 98100 (cf. 82-94 and 122); BENGTSSON, P.A. Two Arabic Versions, $116 \S 3.2 .6$; LEVIN, B. Die griechisch-arabische Evangelien-Übersetzung. Vat Borg. Ar. 95 und Ber orient. Oct. 1108. Uppsala, 1938, 19; CORRIENTE, F. op. cit., 45 § 2.13.2. Véase además CORRIENTE, F. "A Survey of Spirantization in Semitics and Arabic Phonetics», The Jewish Quarterly Review NS 60/2 (1969), 160162. Cf. MONFERRER-SALA, J.P. «Mutatio nominum. Onomástica griega en transcripción árabe», Collectanea Cristiana Orientalia 4 (2007), 83. 


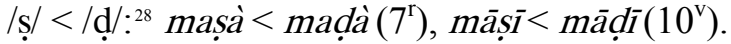

$$
\begin{aligned}
& \text { /ḍ/ </ẓ/:29 hafiụa }<\text { hafiza }\left(2^{\mathrm{r}}\right) \text {. }
\end{aligned}
$$

Como es frecuente, el texto también omite los dos diacríticos suprasegmentales de la tā' marbütah: ${ }^{30}$ al-ihwah < al-ihwat ${ }^{t}\left(2^{\mathrm{r}}\right)$, sanah $<\operatorname{sana}^{t}\left(9^{\mathrm{r}}, 12^{\mathrm{r}}, 15^{\mathrm{r}}, 2^{\mathrm{v}}, 5^{\mathrm{v}}, 7^{\mathrm{v}}, 9^{\mathrm{v}}, 10^{\mathrm{v}}\right.$, $\left.11^{\mathrm{vbis}}, 12^{\mathrm{v}}, 13^{\mathrm{v}}\right)$, miyah $<$ mi' $^{t}\left(10^{\mathrm{r}}, 13^{\mathrm{v}}\right), \operatorname{sab}^{\mathrm{c}} \mathrm{ah}<\operatorname{sab}^{\mathrm{c}}{ }^{t}\left(11^{\mathrm{r}}, 4^{\mathrm{v}}\right)$, sitah $^{\mathrm{sitta}}{ }^{t}\left(11^{\mathrm{r}}, 12^{\mathrm{v}}\right)$, situmiyah $<$ sittumiat $\left(12^{\mathrm{r}}, 2^{\mathrm{v}}, 4^{\mathrm{v}}, 12^{\mathrm{v}}\right)$, arba'amiyah < 'arba'ami'a ${ }^{t}\left(14^{\mathrm{r}}\right)$, dikarah $<$ dikarat $^{t}\left(7^{\mathrm{v}}, 8^{\mathrm{v}}\right)$, talatamiyah $<$ talatami" $^{t}\left(7^{\mathrm{v}}\right)$, miya $<$ mi $^{t}\left(9^{\mathrm{v}}\right)$, hamsah $<$ hamsa $\left(10^{\mathrm{v}}\right)$, talatah $<$ talāta $^{t}\left(12^{\mathrm{v}}\right)$

\section{A) ONOMÁSTICA}

De los nombres propios que encontramos en el texto, salvo uno que procede del griego, el resto son arabizaciones onomásticas de formas antroponímicas hebreas, llegadas al árabe a través del siriaco:

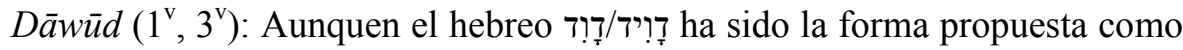
referente de la arabizada Dāwūd ${ }^{31}$, se nos antoja más lógico la transmisión aramea $^{32}$ en función de lo que sucede en mandeo. ${ }^{33}$

Fîlibbus $\left(4^{\mathrm{v}}, 5^{\mathrm{v}}\right)$ : adaptación del siriaco 2 , qua que es la adaptación del griego $\Phi i ́ \lambda \imath \pi \pi \mathrm{s}$.

Isrāîl $\left(14^{\mathrm{r}}, 1^{\mathrm{v}}\right)$ : calco del hebreo \i:ion.

$\operatorname{Yas}_{\bar{u}}$ '( $\left.7^{\mathrm{v}}\right)$ : adaptación del siriaco wan, que es la forma correspondiente del

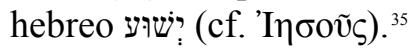

${ }^{27}$ BLAU, J. GCA, 110-111 § 17.2; KNUTSSON, B., Studies, pp. 104-105; BENGTSSON, P.Å. Two Arabic Versions, 122-123 § 3.2.9; CORRIENTE, F. Sketch, 50 § 2.18.2; MONFERRER-SALA, J.P., «Descripción», Collectanea Christiana Orientalia 2 (2005), 117; BLAU, J. Emergence, 229.

${ }^{28}$ Acerca de esta cambio, cf. BLAU, J. GCA, I, 113, n. 176.

${ }^{29}$ BLAU, J. GCA, I, 113-114 $\S \S 20.2-21$; CORRIENTE, F. «A Survey of Spirantization». The Jewish Quarterly Review NS 60/2 (1969), 163. Cf. ARNOLD, Werner. Die arabischen Dialekte Antiochiens. Wiesbaden, 1998, 38.

${ }^{30}$ BLAU, J. GCA, 115-121 $\S ~ 24.1-24.4$; HOPKINS, S. Studies, 44-48 $§ 47$; KNUTSSON, B. Studies, 109-112; BENGTSSON, P.Å. Two Arabic Versions, 124-127 § 3.2.13.

${ }^{31}$ HOROVITZ, Joseph. «Jewish Proper Names and Derivatives in the Koran». Hebrew Union College Annual 2 (1925), 166-167. Cf. NOTH, M. Die israelitischen Personnenamen im Rahmen der gemeinsemitischen Namengebung. Hildesheim, 1966, 183.

${ }^{32}$ JEFFERY, Arthur. The Foreign Vocabulary of the Qur'ān. Baroda, 1938, 128.

${ }^{33}$ DROWER, E. S. y MACUCH, R. A Mandaic dictionary, Oxford, 1963, $98 \mathrm{~b}$.

${ }^{34}$ Sobre la debatida etimología de este nombre, véase NOTH, M. Die israelitischen Personnenamen, 207-209.

${ }^{35}$ MONFERRER SALA, J.P., «Algo más acerca de 'IIsà, el nombre de Jesús en el Islam», Miscelánea de Estudios Árabes y Hebraicos 47/1 (1998), 399-404. Cf. VOLLERS, K., «Ueber die lautliche Steigerung bei Lehnwörtern im Arabischen». Zeitschrift der Deutschen Morgenländischen Gesellschaft 45 (1891), 352. En ocasiones Yasūc $^{\mathrm{c}}$ aparece escrito como Yašŭce véase v.gr. 


\section{B) TOPÓNIMOS}

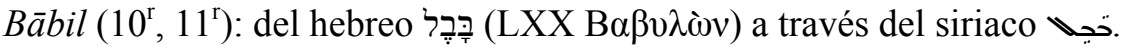

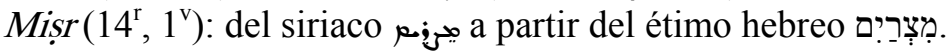

\section{C) PRÉSTAMOS Y NEOLOGISMOS}

Asātīr $\left(7^{\mathrm{r}}\right)$ : término utilizado para referirse a las "narraciones de los antiguos", casi con total seguridad a través del árameo שְָָׁ o del arameo siriaco lida, ${ }^{36}$ ambos con el sentido básico de "escritura; documento" con el que es utilizado entre los cristianos de lengua aramea. ${ }^{37}$

Burğ $\left(10^{\mathrm{r}}, 11^{\mathrm{r}}\right)$ : adaptación del griego $\pi u ́ \rho \gamma o \varsigma$ mediante el cambio $/ \mathrm{b} /</ \pi /$. Iskandariyyīn $\left(4^{\mathrm{v}}, 5^{\mathrm{v}}\right)$ : adaptación del gentilicio griego $\dot{\alpha} \lambda \varepsilon \xi \alpha \nu \delta \rho \varepsilon v ́ \varsigma$ ('alejandrino') a través del topónimo Iskandariyyah $<$ 'A $\lambda \varepsilon \xi \alpha ́ \alpha \delta \rho \varepsilon 1 \alpha$.

$\operatorname{Masīh}\left(7^{\mathrm{v}}, 8^{\mathrm{v}}, 13^{\mathrm{v}}\right)$ : calco del siriaco 20 , מمس Quwwāt $\left(3^{\mathrm{r}}\right)$ : se trata de un neologismo ampliamente atestiguado en la producción árabe cristiana, en sus diversas tradiciones eclesiásticolingüísticas (v.gr. siriaca-árabe o copta-árabe) para referirse al orden angélico de las "virtudes". ${ }^{39}$

Sayyid $\left(6^{\mathrm{v}}, 8^{\mathrm{v}}, 13^{\mathrm{v}}\right)$ : es la traducción-adaptación teológica del griego $\kappa u ́ p r o \varsigma^{40}$

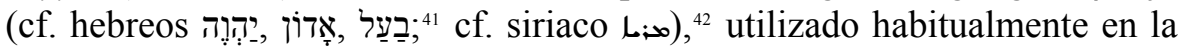
producción de los árabes cristianos en combinación con el término rabb. ${ }^{43}$

MONFERRER-SALA, J.P. Y ZOMEÑO, Amalia. «Abgari regis fabulae versio iuxta narrationem magnae ecclesiae Edessae aedificationis (secundum Sinaiticum arabicum CDXLV qui in Coenobio Sanctae Catalinae asservatur)». Collectanea Christiana Orientalia 5 (2008), $162 \S 5.1$.

${ }^{36}$ JEFFERY, A., The Foreign Vocabulary of the Qur'an, p. 56.

${ }^{37}$ MONFERRER SALA, J. P., «Gharshunitica. Abgar and Jesus' Letters in the Arabic version of Michael the Syrian's Chronicle (Brit. Mus. Or. 4402 ff $55^{\mathrm{V}}-56^{\mathrm{r}}$ )». En. Orientalia Christiana. Festschrift für Hubert Kaufhold zum 70. Geburtstag. Ed. Peter Bruns y Heinz Otto Luthe. Wiesbaden, 2013, p. 299 § XII.

${ }^{38}$ Sobre el término al-Masīh en árabe, véase GRAF, Georg. «Wie ist das Wort al-Masîh zu Übersetzen?». Zeitschrift der Deutschen Morgenländischen Gesellschaft 104 (1954), 119-123.

${ }^{39}$ MONFERRER-SALA, J. P., «The creation of seven archangels and their hosts in a Coptic-Arabic Hexaemeron attributed to Epiphanius of Cyprus». Journal of Coptic Studies 14 (2012), pp. 72-73 §6.

${ }^{40} \mathrm{Cf}$. MOULTON, J.H. y MILLIGAN, G. The vocabulary of the Greek Testament illustrated from the papyri and other non-literary sources. London, 1914-1929, 365a-366b.

${ }^{41}$ Cf. ABBOTT-SMITH, G. A Manual Greek Lexicon of the New Testament. Edinburgh - New York, 2005 (= 1936), 261-262.

${ }^{42}$ Cf. MONFERRER-SALA, J.P., «A Nestorian Arabic Pentateuch used in Western Islamic Lands», en The Bible in Arab Christianity. Ed. David THOMAS. Leiden - Boston, 2007, 367-368.

${ }^{43}$ Cf. MONFERRER-SALA, J.P. «Estrategias de traducción en un texto árabe cristiano con Vorlage siriaca ApVir(ar) 4-5 [ApVir(sir) 5-6]». Estudios de dialectología norteafricana y andalusi 12 (2008), 24, 32-33. 


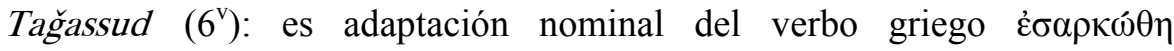
('encarnó'), no referido al nacimiento propiamente dicho, sino a la concepción de Jesús en el seno de María la Virgen.

Yūnānī $\left(4^{\mathrm{v}}, 6^{\mathrm{v}}\right)$ : calco del griego í $\omega v$ (cf. ióvios), 'jonio'. ${ }^{44}$ La alusión a Filipo II como yūnānī, es decir como 'jonio', aunque más probablemente como 'griego', pudiera deberse a una confusión con la ocupación de Jonia por el ejército macedonio de su hijo Alejandro Magno tras el domino persa aqueménida de esa región. ${ }^{45}$

\section{D) TERMINOLOGÍA CRONOLÓGICA}

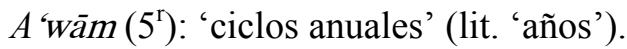

Ayyām (5 $\left.5^{\mathrm{r}}\right)$ : 'días'.

Hisāâ $\left(1^{\mathrm{I}}\right)$ : 'cómputo'.

Marra-yamurru (4'): 'transcurrir'.

Qamar (4): 'luna'.

Šams (4): 'sol'.

$\operatorname{Sinūn~(4'):~'años'.~}$

Šuhūr (5): 'meses'.

Tawārīhn $\left(1^{\mathrm{r}}\right)$ : 'eras'.

\section{EDICIÓN DIPLOMÁTICA DEL TEXTO ÁRABE}

En la edición del texto árabe seguimos el criterio de ofrecer la lectura del texto tal cual éste aparece en el manuscrito, respetando las peculiaridades tanto ortográficas como lingüísticas que presenta, con el fin de que los interesados puedan disponer de una edición fiel del texto tal cual la fijó el copista.

En nuestra edición hemos mantenido la disposición linear del texto original con el fin de facilitar las referencias en el apartado del estudio paleográfico y lingüístico previo.

RECTO

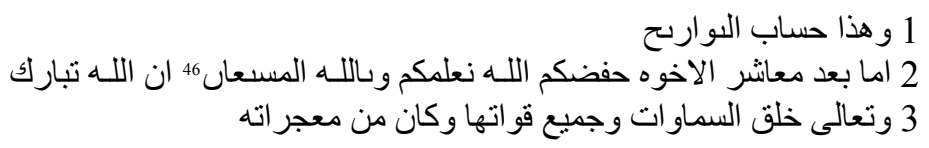

${ }^{44}$ LIDDELL, Henry George y SCOTT, Robert. A Greek-English Lexicon. New York - Chicago, 1897 ( $8^{\mathrm{a}}$ ed.), $717 \mathrm{~b}$.

${ }^{45} \mathrm{Cf}$. el título de 'rey de Macedonia' que se infiere de la oración de malaka 'alà Maqdūniyyah ('reinó sobre Macedonia') que se le da en el Kitāb al-'Unwān: VASILIEV, Alexandre, "Kitab al“Unvan. Histoire universelle écrite par Agapius (Mahboub) de Menbidj”. En Patrologia Orientalis, ed. R. GRAFFIN - F. NAU. Paris, 1910, V, 92 [220].

ha sido escrito sobre la línea de escritura, en $1^{r}$, a continuación del título por olvido del copista. 


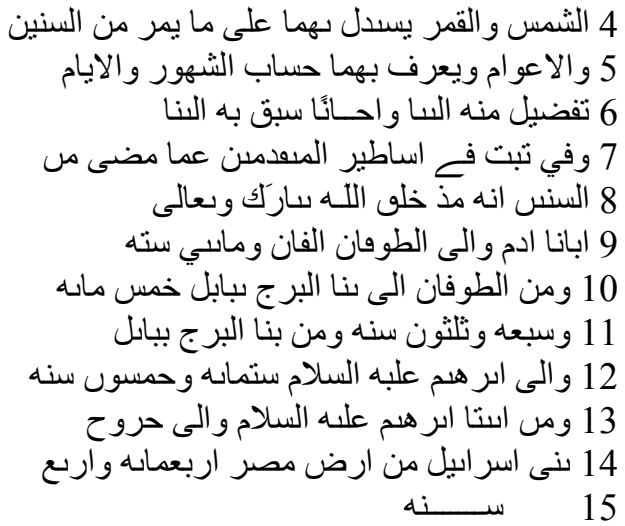

\section{VERSO}

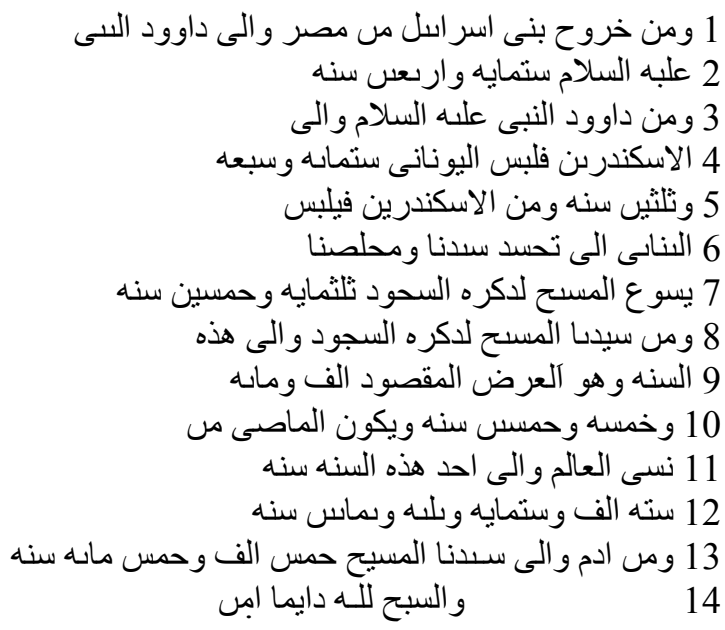

\section{TRADUCCIÓN}

\section{RECTO}

1. Este es el cómputo de las eras.

2. ¡Hermanos, Dios preserve vuestro saber, encomendaos a Dios!, pues Dios, bendito

3. y altísimo, creó los cielos y todas sus potencias. Entre sus prodigios se hallan

4. el sol y la luna, con los que se sigue el transcurso de los años

5. y los ciclos anuales, mediante los que se reconoce el cómputo de los meses y de los días,

6. como ayuda y beneficio que tuvimos con anterioridad.

7. En (la) relación de (las) historias antiguas que transcurrieron desde

8. los años desde que Dios, bendito y altísimo, creó 
9. a nuestro padre Adán hasta el Diluvio hay dos mil doscientos años.

10. Desde el Diluvio hasta la construcción de la torre en Babel quinientos

11. treinta y siete años. Desde la construcción de la torre en Babel

12. hasta Abraham, la paz sea con él, seiscientos cincuenta años

13. Desde los hijos de Abraham, la paz sea con él, hasta el Éxodo

14. de los hijos de Israel del país de Egipto cuatrocientos cuarenta

15. años.

VERSO

1. Desde el Éxodo de los hijos de Israel de Egipto hasta el profeta David,

2. la paz sea con él, seiscientos cuarenta años.

3. Desde el profeta David, la paz sea con él, hasta

4. los alejandrinos, (hasta) Filipo el griego ${ }^{47}$ seiscientos treinta

5. y siete años. Desde los alejandrinos, (desde) Filipo

6. el griego, hasta la encarnación de nuestro Señor y Salvador nuestro,

7. Jesús el Mesías, cuya memoria adoramos, trescientos cincuenta años.

8. Desde nuestro Señor el Mesías, cuya memoria adoramos, hasta este

9. año, que es la extensión referida, mil ciento

10. cincuenta y cinco años. Han transcurrido desde

11. la formación del mundo hasta el final de este año, año

12. seis mil seiscientos ochenta y tres años

13. y desde Adán hasta nuestro Señor el Mesías cinco mil quinientos años

14. Alabado sea Dios por siempre. Amén

\section{NOTAS AL TEXTO}

Aunque el texto no indica las referencias concretas de los pasajes a los que alude como hitos referentes de los cómputos cronológicos que ofrece, estos se infieren claramente de los enunciados, que relacionamos a continuación con sus correspondientes referencias bíblicas:

$\begin{array}{llll}8^{\mathrm{r}}-9^{\mathrm{r}} & \text { Creación de Adán } & \rightarrow & \text { Gn 1,27 } \\ 9^{\mathrm{r}}, 10^{\mathrm{r}} & \text { Diluvio } & \rightarrow & \text { Gn 7,10-8,2 } \\ 10^{\mathrm{r}}, 11^{\mathrm{r}} & \text { Construcción torre Babel } & \rightarrow & \text { Gn 11,3-8 } \\ 12^{\mathrm{r}} & \text { Abraham } & \rightarrow & \mathrm{Gn} 11,26 \\ 13^{\mathrm{r}} & \text { Hijos de Abraham } & \rightarrow & \mathrm{Gn} 16,4 \\ 13^{\mathrm{r}}, 14^{\mathrm{r}} & \text { Éxodo } & \rightarrow & \mathrm{Ex} 1,1\end{array}$

${ }^{47}$ Se trata de Filipo II (382-336 a.C.), rey de Macedonia y padre de Alejandro Magno, citado en 1 Macc 1,1 y 6,2. Sobre este monarca, véase BRADFORD, Alfred S. Philip II of Macedon: A Life from the Ancient Sources. Westport CT, 1992. 


$\begin{array}{llll}1^{\mathrm{v}} & \text { Éxodo de los hijos de Israel } & \rightarrow & \text { Ex 1,1 } \\ 1^{\mathrm{v}}, 3^{\mathrm{v}} & \text { El profeta David } & \rightarrow & 2 \text { Sam 2,1 } \\ 4^{\mathrm{v}}, 5^{\mathrm{v}}, 6^{\mathrm{v}} & \text { Filipo el Griego } & \rightarrow & 1 \text { Mac 1,1;6,2 } \\ 6^{\mathrm{v}}, 7^{\mathrm{v}} & \text { Encarnación de Jesús } & \rightarrow & \text { Mt 1,25 (par. Lc 2,7) }\end{array}$

Por su parte, las correspondencias entre las referencias bíblicas y los cómputos cronológicos que ofrece el autor para el tiempo transcurrido entre cada dos referencias son las siguientes:

Creación de Adán $\rightarrow$ Diluvio

Diluvio $\rightarrow$ construcción de la torre en Babel

Construcción de la torre en Babel $\rightarrow$ Abraham

Hijos de Abraham $\rightarrow$ Éxodo

Éxodo $\rightarrow$ David

David $\rightarrow$ Felipe el Griego

Felipe el Griego $\rightarrow$ nacimiento de Jesús

Nacimiento de Jesús $\rightarrow$ año 1233

Creación del mundo $\rightarrow$ año 1233

Creación de Adán $\rightarrow$ nacimiento de Jesús

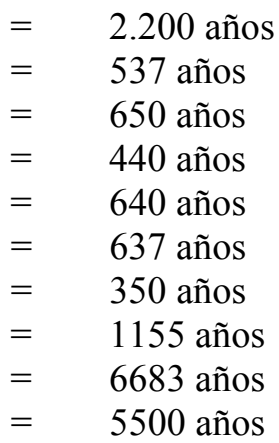

Como hemos indicado en la introducción, los dos principales métodos de cómputo, el alejandrino y el bizantino mediaban aproximadamente unos 17 años en el año computacional de inicio. Del mismo modo, los cálculos no sólo no coinciden con la compleja cronología que exhiben los textos bíblicos, ${ }^{48}$ con los que tampoco coinciden las cifras que ofrece nuestro autor melkita: v.gr. 2200 años que no coinciden exactamente con la genealogía de los setitas que presenta Gn 5,25-32 a la que sin duda sigue para establecer el cómputo entre la creación de Adán y el comienzo del diluvio; ${ }^{49}$ o los 537 años, que tampoco coinciden con Gn 11,10-26 que recoge la genealogía de Sem entre el diluvio y Abraham..$^{50}$

Sin embargo, los ciclos y los cómputos tampoco equivalen a otros elaborados por autores melkitas, como es el caso del célebre cronógrafo Agapio de Menbiğ ${ }^{51}$ quien en su Kitāb al-unwān ofrece una cronología del mundo hasta la época de Moisés, que no coincide con la de nuestro autor: v.gr. mientras nuestro autor da un

48 MONFERRER-SALA, J.P. «Languages and texts: A summary in Michael the Syrian's 'Chronography' and its references in Greek, Syriac and Arabic, with an incursion in Ethiopic». En prensa.

${ }^{49}$ Véase al respecto HUGHES, Jeremy. Secrets of the Times: Myth and History in Biblical Chronology. Sheffield, 1990, 5-30, 43-54.

${ }^{50}$ HUGHES, J. Secrets of the Times, 30-43, 43-54.

${ }^{51}$ Sobre Mạ̣būb ibn Qustanțīn al-Rūmī al-Manbijī y su obra, véase GRAF, G., Geschichte der christlichen arabischen Literatur. Città del Vaticano, 1947 (reed. Modena, 1996), II, 39-41; SWANSON, Mark N., «Maḥbūb ibn Qusțanțin al-Manbijī». En Christian-Muslim Relations. A Bibliographical History. Volume 2 (900-1050). Ed. David THOMAS et al. Leiden - Boston, 2010, 241-245. 
cálculo de 2.200 años para el periodo que media entre la creación del mundo y el comienzo del diluvio, sin embargo Agapio de Menbiğ ofrece un total de 2256, con una diferencia, por lo tanto, de 56 años, ${ }^{52}$ que es el cómputo que recoge, v.gr., el cronógrafo siriaco occidental Miguel el Sirio para este mismo periodo. ${ }^{53}$

Con todo, la tabla que presenta Miguel el Sirio en la que establece el cómputo desglosado de años que lleva desde Adán hasta el nacimiento de Abraham el proceso de desglose es distinto al que presenta Agapio de Menbiğ y exhibe una diferencia que se reduce a 30 años con respecto al cómputo que da nuestro autor melkita en el texto arriba editado. ${ }^{54}$

${ }^{52}$ VASILIEV, A. «Kitab al-'Unvan. Histoire universelle écrite par Agapius (Mahboub) de Menbidj». En Patrologia Orientalis. Ed. R. GRAFFIN - F. NAU. Paris, 1910, V, 680-682 [124-126], cf. para un periodo posterior 688-689 [132-133].

${ }_{53}$ Chronique de Michel le Syrien, Patriarche jacobite d'Antioche (1166-1199). Edición y traducción francesa de Jean-Baptiste CHABOT. 4 vols. Paris, 1899, 1901, 1905, 1910, I, 13, 28. Sobre este autor y su obra, véase VAN GINKEL, Jan. "A man is not an island. Reflections of the historiography of the early Syriac Renaissance in Michael the Great". En The Syriac Renaissance. Ed. Herman TEULE et al. Louvain, 2010, 113-121. Cf. Ignatius Aphram Barsoum. The Scattered Pearls. Piscataway NJ, 2003 (2 ed.), 445-448, y DUVAL, Rubens. La littérature siriaque. Paris, 1907, 196198. Sobre el texto de esta cronografía, véase BROCK, Sebastian P. "Syriac historical writing: a survey of the main sources". Journal of the Iraqi Academy [sección siriaca] V (1979-1980), 309.

${ }^{54}$ Chronique de Michel le Syrien. Ed. y trad. J.-B. CHABOT, I, p. 28. Cf. VASILIEV, A. "Kitab al-'Unvan”, en Patrologia Orientalis. Ed. R. GRAFFIN - F. NAU, V, 680 [124]. 